\title{
Démêler les lignes : carnets et croquis préparatoires
}

Sabine Teyssonneyre

\section{Q OpenEdition}

1 Journals

\section{Édition électronique}

URL : http://journals.openedition.org/genesis/1696

DOI : 10.4000/genesis. 1696

ISSN : 2268-1590

Éditeur :

Presses universitaires de Paris Sorbonne (PUPS), Société internationale de génétique artistique littéraire et scientifique (SIGALES)

Édition imprimée

Date de publication : 12 décembre 2016

Pagination : 111-120

ISBN : 9791023105490

ISSN : 1167-5101

\section{Référence électronique}

Sabine Teyssonneyre, «Démêler les lignes : carnets et croquis préparatoires », Genesis [En ligne], 43 | 2016, mis en ligne le 29 octobre 2017, consulté le 19 avril 2019. URL : http:// journals.openedition.org/genesis/1696; DOI : 10.4000/genesis.1696

Ce document a été généré automatiquement le 19 avril 2019.

Tous droits réservés 


\title{
Démêler les lignes : carnets et croquis préparatoires
}

\author{
Sabine Teyssonneyre
}

1 Le carnet est un outil quasi indispensable pour beaucoup d'auteurs ou de théoriciens, bien que les usages que l'on puisse en faire soient très différents. On peut décider de séparer strictement le texte du dessin, la pensée de l'image, ou les mêler et de ce fait mélanger leurs natures. Le carnet est un lieu intime où l'on peut faire erreur et ou l'on est loin du regard. C'est un bon endroit pour expérimenter et commencer à définir des territoires de pensée et de dessin. C'est aussi dans ces carnets que s'élabore le langage du dessinateur: toute une batterie de traits, de formes et de contre-formes, des tics de dessin et des reprises qui vont former une sorte de grammaire visuelle en formation. Le carnet de croquis permet d'avoir des dessins qui ne sont pas sacralisés par un cadre, une impression en fanzine ou une publication sur Internet. De ce fait, ils sont manipulables, et peuvent être retravaillés, joints entre eux, et souvent abandonnés en route. Ils constituent une sorte de terreau pour les œuvres abouties à venir, même s'ils ne sont pas directement des projets en devenir. Il s'agit d'exercices, de tessons d'inconscient et de pépites dont personne ne sait encore s'ils vont être un jour ou non exposés aux regards.

2 Le croquis ou l'écriture se pensent en même temps qu'ils se construisent. À ce stade du processus, la différence n'est pas encore très nette entre l'un et l'autre. La ligne n'a pas encore trouvé son genre, les ratures et les urgences laissent l'écriture être dessin et le croquis notation. Il se pourrait qu'ils sortent de la même matière. Des lignes emmêlées qui doivent se déplier, qui ne savent pas encore ce qu'elles sont mais savent ce qu'elles veulent. 


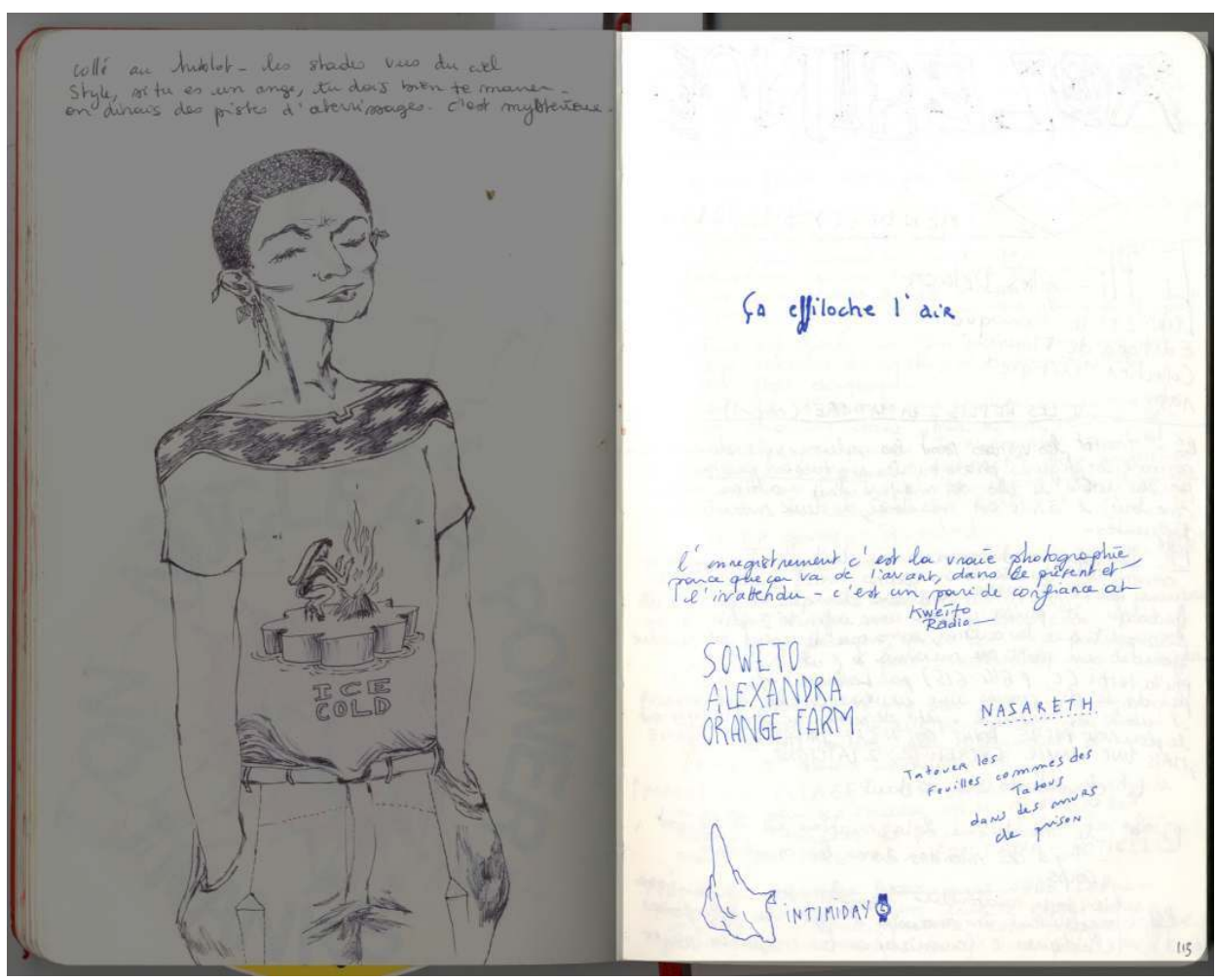

Fig. 1 : Exemple de page où le texte et le dessin sont très proches. II y a une composition spatiale et différentes sortes d'écritures, qui traduisent des natures différentes de l'idée. C'est une pensée associative : on voit le fantôme accompagné de la mention « Intimiday » et une montre. Cet assemblage fonctionne comme un rébus. II y a une intuition, entre intimité, temps et « intimidé », les draps froissés du fantôme, ce qu'il cache, l'idée de day et de montre, et tous ces sens s'emmêlent et résonnent entre eux. Ce sont des concepts à la lisière de l'informulable, mais ils trouvent une certaine stabilité dans cette notation. II s'agit presque d'une sensation.

On peut remarquer cette annotation: «tatouer les feuilles comme des tatous dans les murs des prisons ". Il y a aussi cette idée d'archéologie, de territoire de l'idée : elle se déploie sur la feuille en giclures et c'est à celui qui les relira (en l'occurrence moi) de mettre un peu d'ordre dans tout cela.

Souvent, les recherches et les études au sein des carnets comprennent des éléments que l'on connaît déjà, des tournures de phrases ou des traits de dessin que l'on a déjà utilisés et qui relèvent presque de l'artisanat - si l'on peut considérer que l'art contient la surprise et le vertige, et que l'artisanat s'emploie à reproduire les formes qui en sont issues, les vidant peu à peu de leur sens.

Un jour, je marchais avec une amie dans un endroit dangereux. J'ai commencé à marcher devant parce que je devais me concentrer sur tous les dangers possibles, et ma concentration a évacué ma peur. J'ai parfois l'impression qu'écrire ou dessiner (faire un croquis) fonctionne comme notre marche de cette nuit-là. Il faut avancer sans être sûre de ce qui est en train de se passer et atteindre un état d'aveuglement où le sens et la sensation s'emmêlent. Thésée dans le labyrinthe déroule le fil d'Ariane dans un domaine inconnu. Notre fil, c'est la ligne. La ligne de texte et celle du croquis tracent leur chemin dans le labyrinthe en même temps qu'elles l'empruntent. À ce moment-là, les outils dont on dispose (le carnet, le stylo) sont comme une extension du moi et on est au plus près de la sensation de dessin, ou de la sensation de pensée. On fait corps avec ses outils de façon à les évacuer en tant que biais. Dans le moment du croquis, des associations d'idées peuvent nous lancer sur un milliard d'autres voies, et il faut choisir celles qu'on 
emprunte, mémoriser des fragments d'idées pour les réutiliser quelques secondes plus tard et toujours tenir cette pensée ou ce croquis pendant le moment de son élaboration, sinon elle s'évapore et les lignes tracées deviennent des choses mortes.

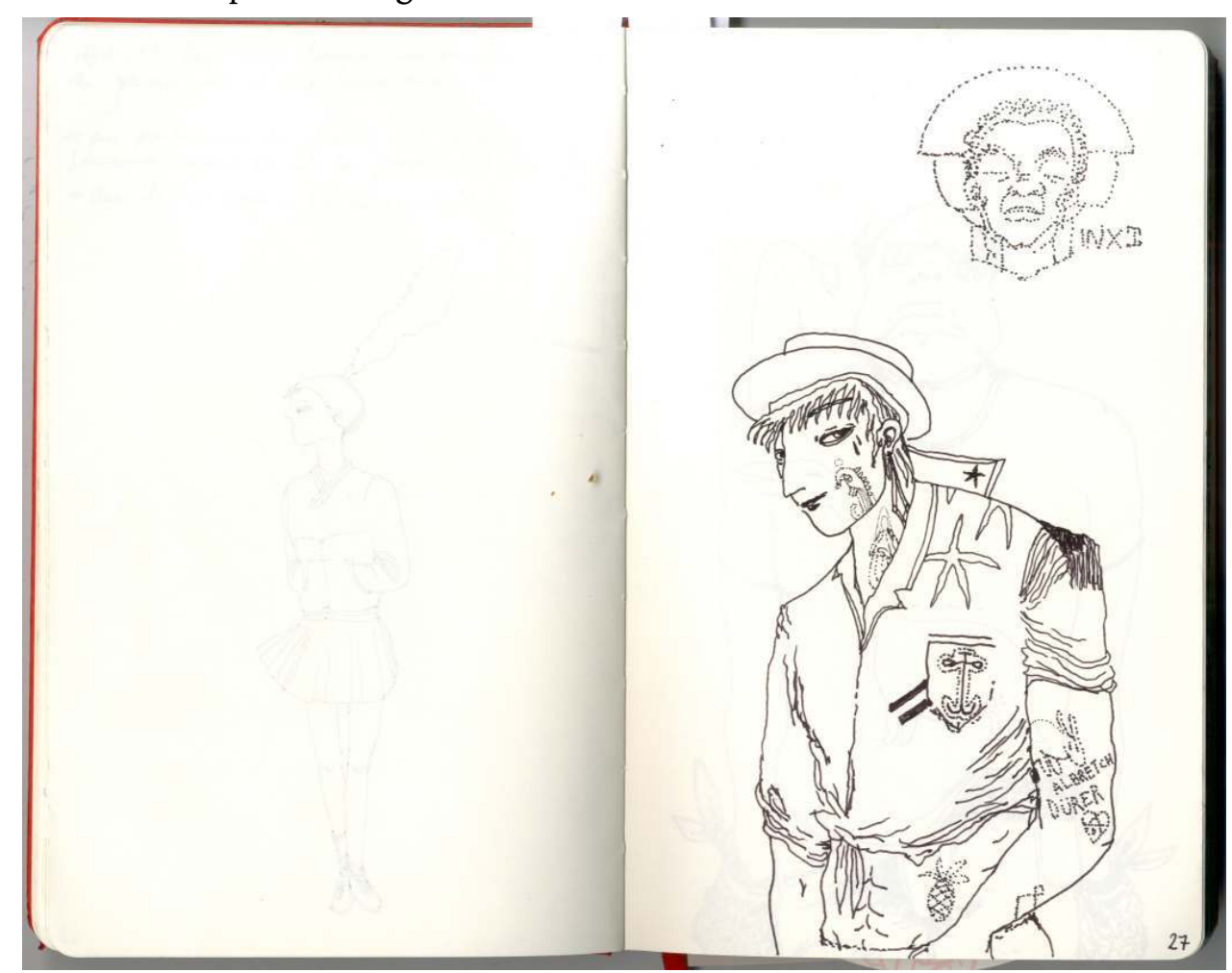

Fig. 2

6 Ce dessin (fig. 2) reprend des choses que je sais déjà faire : des tatouages, un marin... Le trait est déjà mort parce que je me désintéresse de ce dessin en même temps que je le fais. Il peut être qualifié d'artisanat parce qu'il ne produit rien de neuf, il n'y a aucune tentative là-dedans. Il ne fait que reprendre des techniques déjà éprouvées, en les vidant de leur raison d'être et de leur justesse. Ce mimétisme de la forme n'a pas d'intérêt si on veut être juste : il sert simplement à développer un confort visuel, d'éléments connus et inoffensifs.

7 Au contraire, je ne sais pas faire de manga et de trait simple, l'outil est inconnu (fig. 3) : il s'agit du même stylo-plume mais utilisé à l'envers de la pointe pour déposer moins d'encre et obtenir un trait plus fin. Ces deux composantes inconnues (l'outil et le sujet) m'obligent à rester en éveil et je trouve qu'il y a ici plus de vigueur et de force dans le placement des lignes. Même si certaines proportions sont fausses, l'ensemble est tenu par une espèce d'équilibre qui résulte de la tension entre moi et le dessin. 


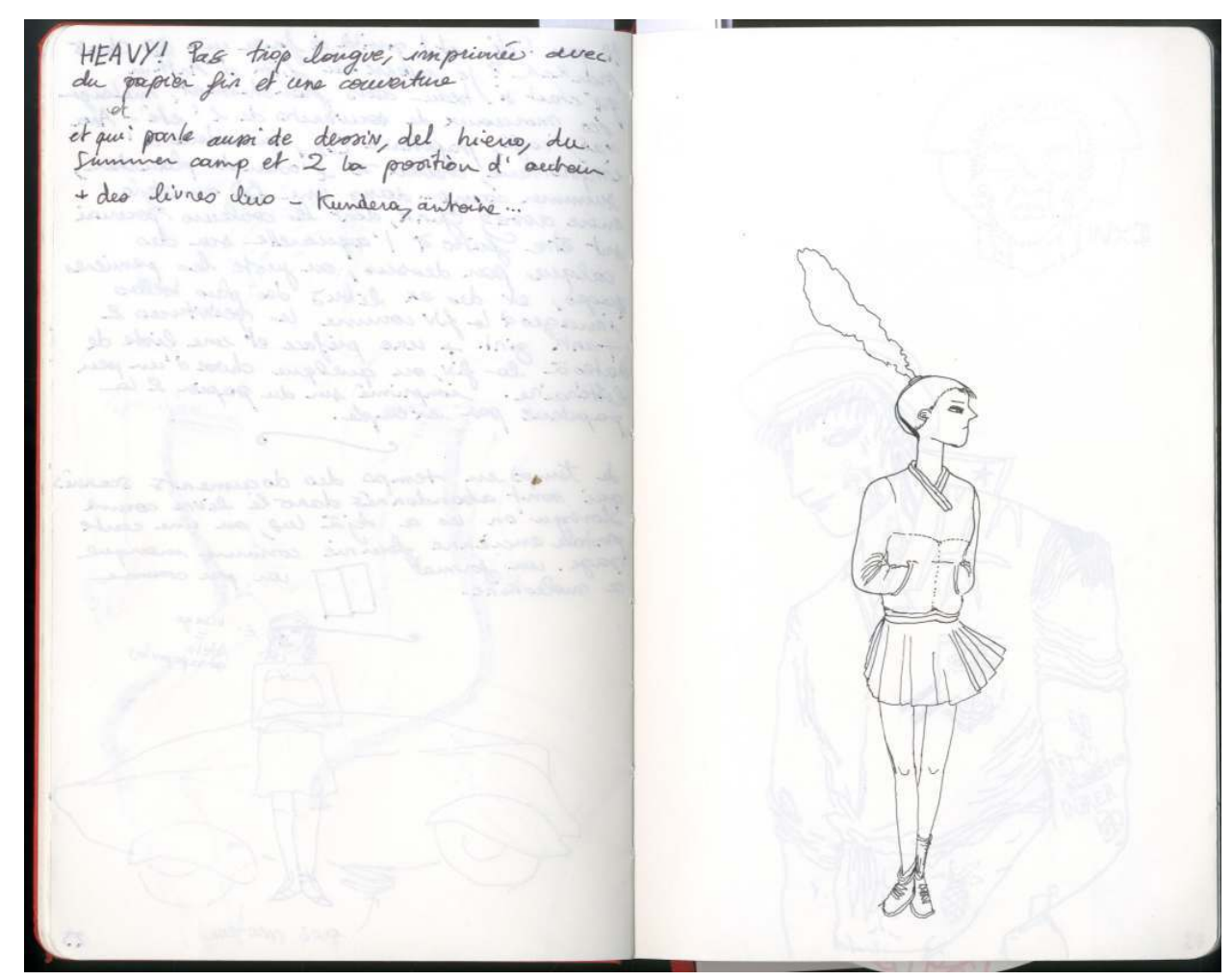

Fig. 3

8 Le croquis ou la note sont dans le moment de leur exécution un actif explosif et instable comparable à la nitroglycérine, car leur nature est encore si inconsistante qu'elle peut basculer d'un instant à l'autre. La moindre perturbation peut être mise à profit. Il s'agit presque d'une chronophotographie de la pensée.

9 Une fois finie la phase d'écriture, il reste ces traits sur le sol de la page, comme le fil d'Ariane en vue aérienne ou comme des draps froissés après une nuit intense - c'est selon. La pensée indéterminée a pu prendre une forme, avec pas mal de hasard et quelques décisions cruciales. Ce qu'on a sous les yeux sont ses premiers pas, mais si on les laisse dans cet état ils ne seront jamais utiles. Le croquis est une façon de donner forme à l'intuition pour la rendre manipulable.

10 «Ainsi des écritures de [Cy Twombly]. Ce sont des bribes d'une paresse, donc d'une élégance extrême; comme si, de l'écriture, acte érotique fort, il restait la fatigue amoureuse : ce vêtement tombé dans un coin de la feuille ", dit Barthes à propos des œuvres de Twombly ${ }^{1}$. Les croquis et les premières écritures me font parfois penser à cela. Les vêtements froissés sont si beaux qu'il peut être tentant de les laisser comme tels. Mais c'est un piège qu'il faut dépasser, car si on donne trop d'importance aux croquis, on ne dépasse jamais la phase embryonnaire de la création. La fascination pour cette sauvagerie et cette liberté ne doit pas nous coincer dans des états où tout est indéterminé, - donc où tout est possible. Il est très dangereux de se laisser aller à la paresse, et de se satisfaire des premiers jets et des croquis préparatoires, parce qu'ils laissent la création ouverte. Leur qualité évidente est d'être un premier choix sur une longue série de décisions qui vont raffiner la pensée. Ils peuvent être beaux tel quel, mais c'est en les retravaillant et en les abandonnant qu'on peut aboutir un projet. Une fois que l'on s'est détaché de ces premiers jets de pensée et qu'on a construit une œuvre finie, on peut y revenir. Ils pourront alors être fascinants, d'autant plus qu'ils auront donné quelque chose. 
11 Les croquis sont comme les photos de jeunesse où de jeunes garçons effrayent la pellicule avec leurs cheveux trop emmêlés par le soleil. Ils documentent les origines d'une œuvre mais ne lèvent pas le mystère de l'essence de l'art.

\section{Naissance d'une écriture}

Cet été, j'ai lu Le Pli de Gilles Deleuze ${ }^{2}$. Les idées de ce livre ont profondément influencé mon dessin. En fait il s'agit presque de l'acquisition progressive d'un nouveau langage. Tout cela s'est passé par une suite de hasards, de contraintes techniques et de décisions plastiques que je n'avais pas conscience de prendre. Au début de l'été, j'ai achetê ce moleskine rouge. La qualitê du papier très lisse m'oblige très vite à trouver un outil qui ne s'effacerait pas. Un crayon n'aurait pas suffisamment accroché le papier et aurait fini par se décoller de la page et disparaître. Pour des raisons pratiques, j'ai donc choisi un bic noir, que je pouvais emmener partout sans craindre une fuite d'encre et qui était beaucoup plus neutre techniquement qu'un pinceau ou une craie grasse : avec ce bic je pourrais à la fois écrire et dessiner. Deux formes de langage vont alors s'influencer et se mixer en une nouvelle pratique du dessin.

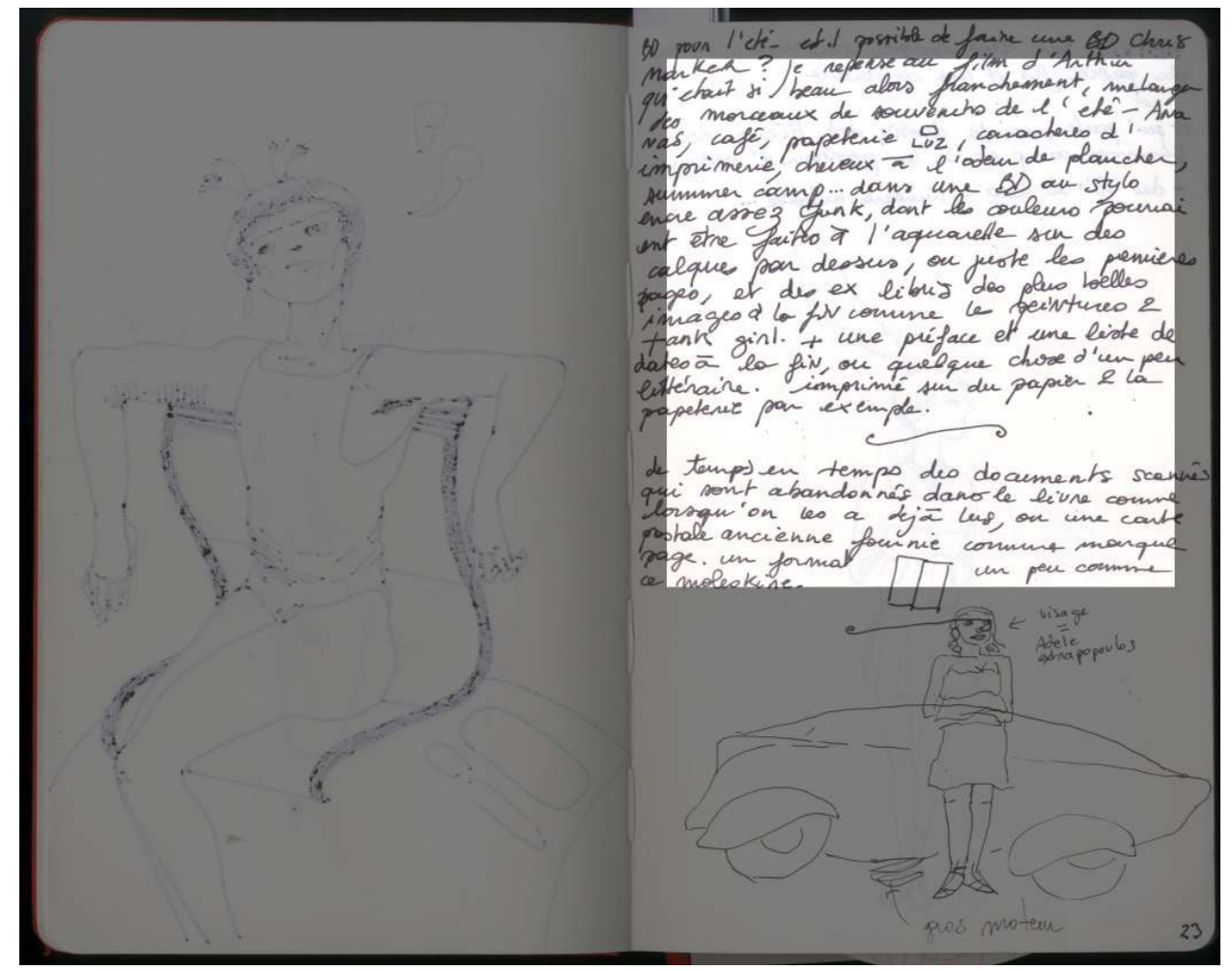

Fig. 4 : Le carnet n'est pas encore apprivoisé, je n'ai pas encore le bon outil. II s'agit d'un stylo encre. II bave trop et traverse les pages : je vais vite abandonner cet outil. 


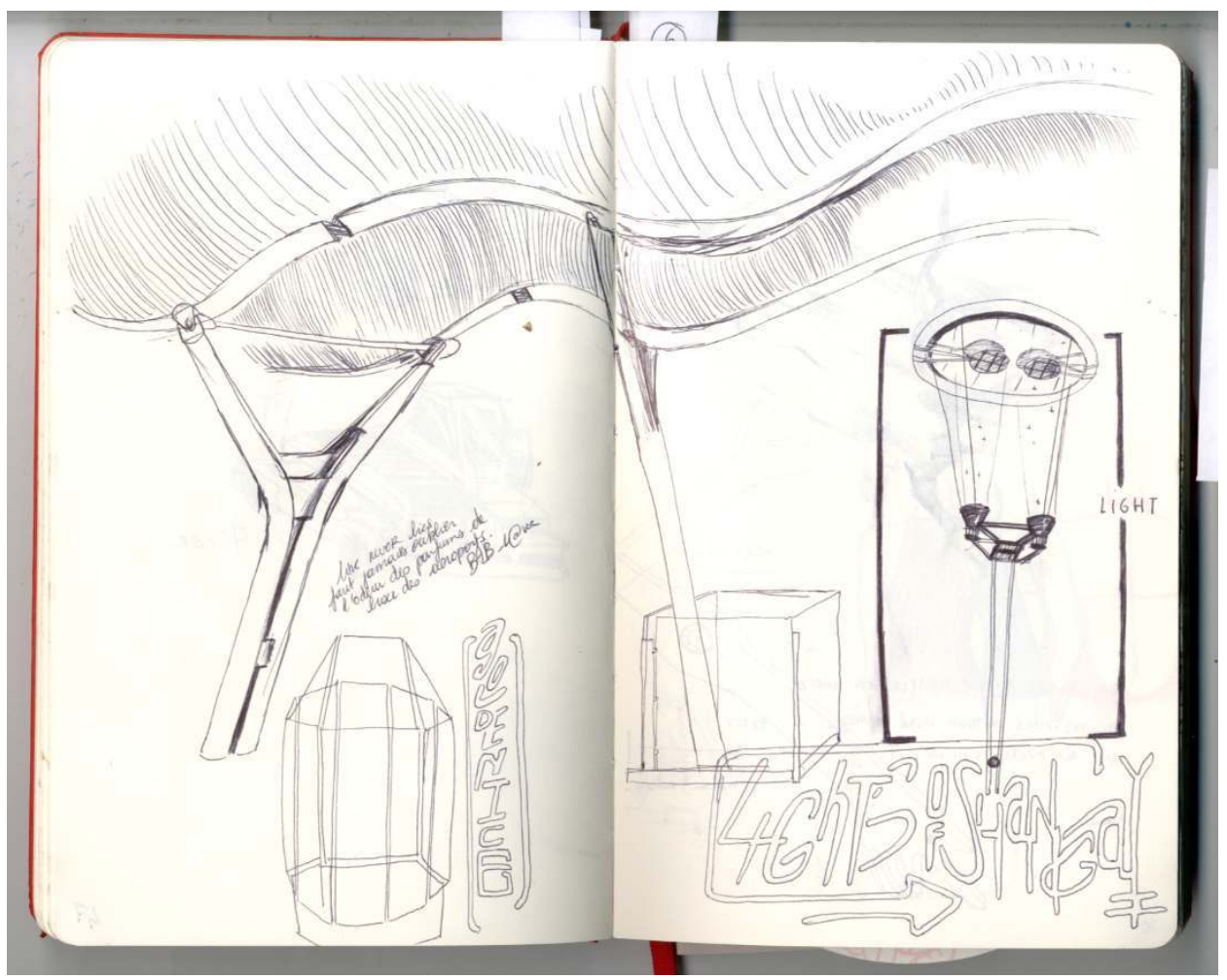

Fig. 5 : Il s'agit de l'aéroport de Madrid. On peut voir que j'essaye de mettre à profit la contrainte d'une pointe sèche pour apprivoiser des lignes plus précises et plus fines. Ici le texte et le dessin se mélangent. « LIGHT » devient un élément du croquis et on peut voir en dessous un texte dessiné. À gauche, le petit texte est encore une autre façon de dérouler cette ligne de Bic. 


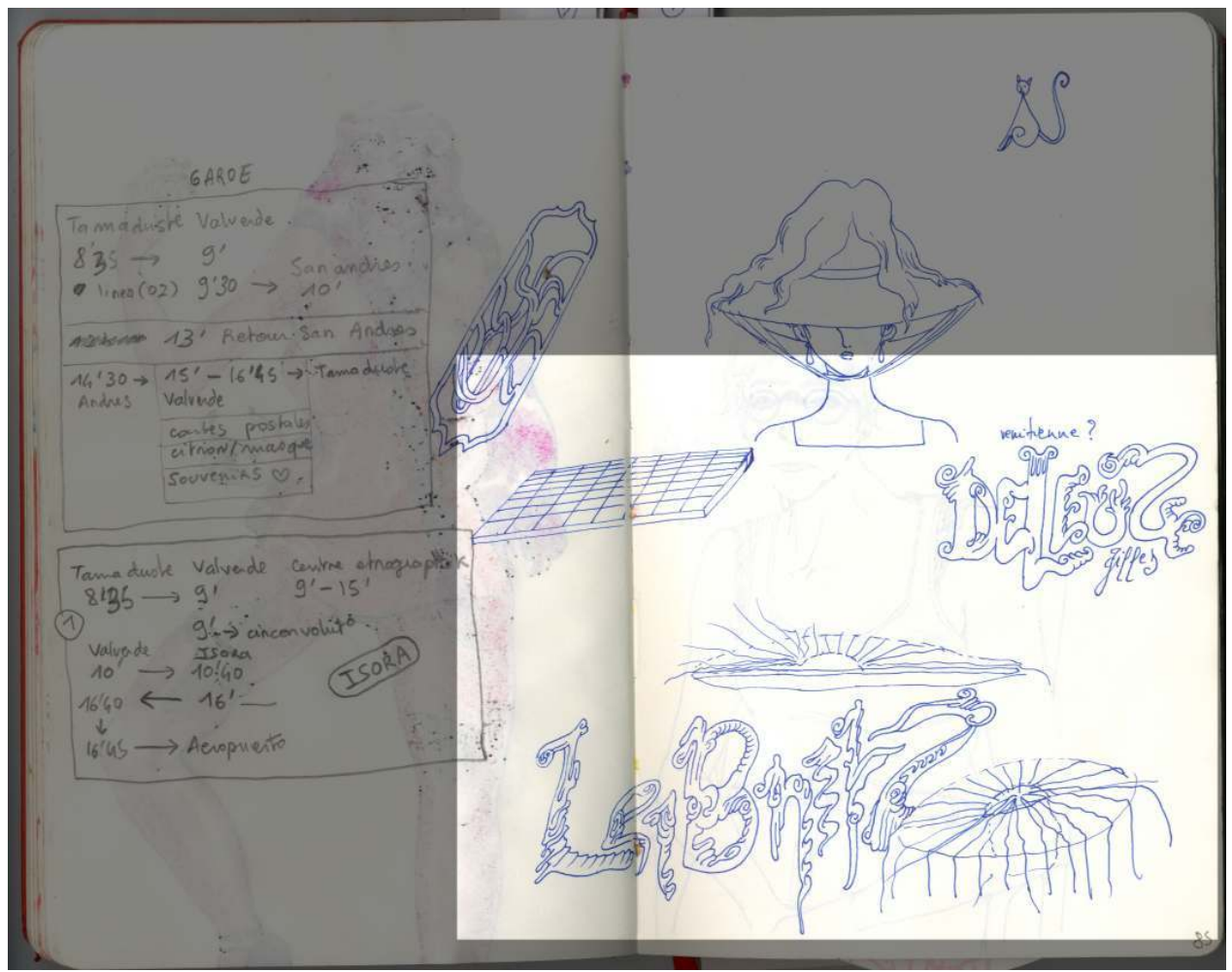

Fig. 6 : Première apparition de Deleuze et Leibniz. À ce moment, je ne comprenais pas encore l'idée du baroque et du pli ; il s'agit juste d'une idée qui commence à prendre forme. C'est la première fois que j'expérimente ce type de ligne plissée. La ligne cherche à comprendre l'idée en se déroulant, comme le fil d'Ariane pour se repérer dans le labyrinthe. Cette ligne qui met du temps à se dessiner est aussi un moyen de compréhension, elle deviendra presque par la suite une transe de scribe qui permet de rester dans cet état d'éveil et d'intuition pour garder le dessin vivant. 


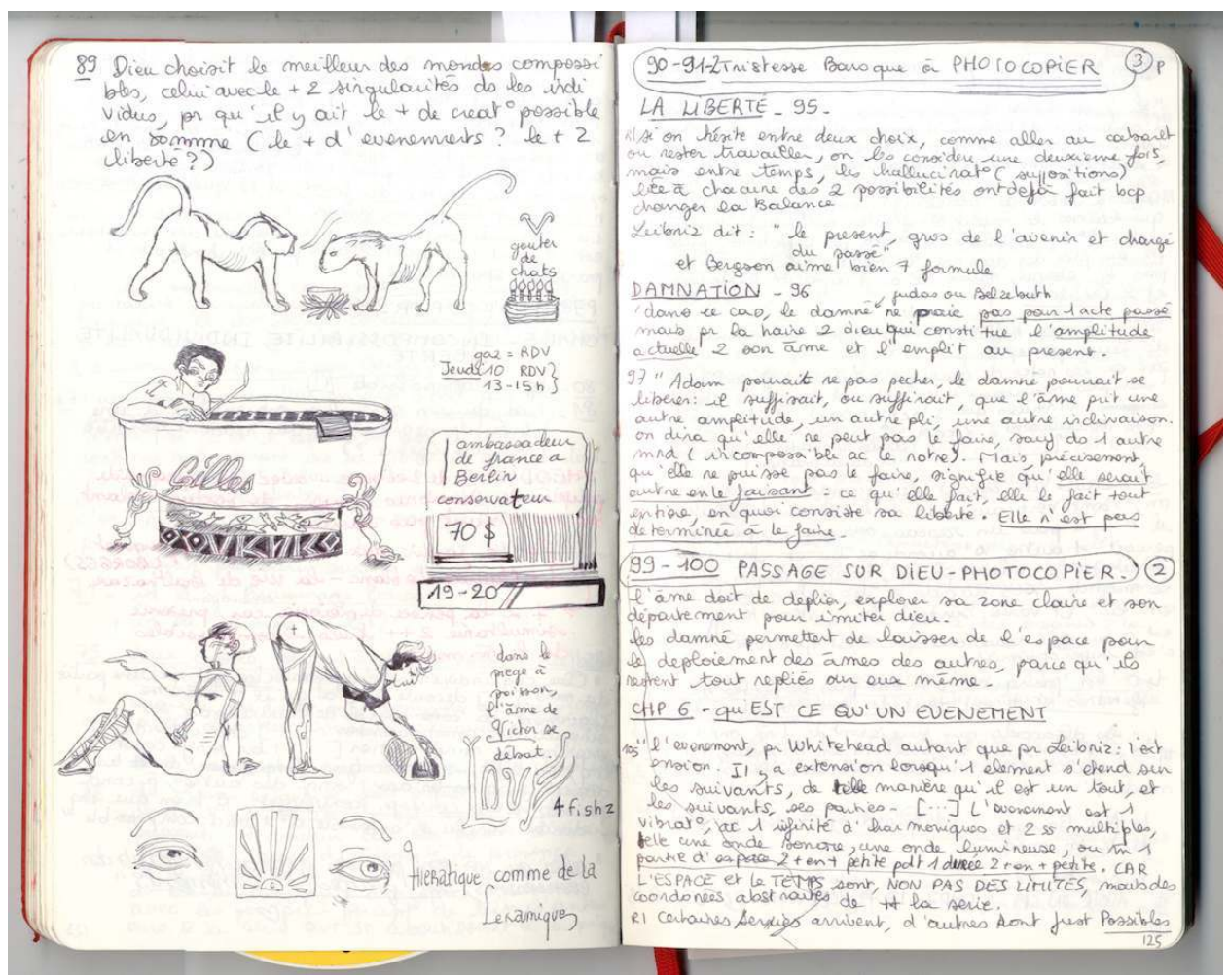

Fig. 7 : Ici, une page sur laquelle je recopie des passages de Pli et des réflexions associées pour les stocker en vue de les réutiliser plus tard. L'idée de stockage de pensée est très importante, et très liée à celle du pli baroque. L'outil Bic permet d'écrire très petit et de compresser les idées en un tissage très serré. Dans cette forme d'écriture commence un apprentissage parallèle : celui d'une finesse et d'une précision de plus en plus extrême. En effet, mon écriture va être de plus en plus dense au moment où mon trait de dessin va s'affiner et s'ourdir de volutes et de replis intérieurs. On peut voir à gauche Gilles Deleuze dans sa baignoire. 


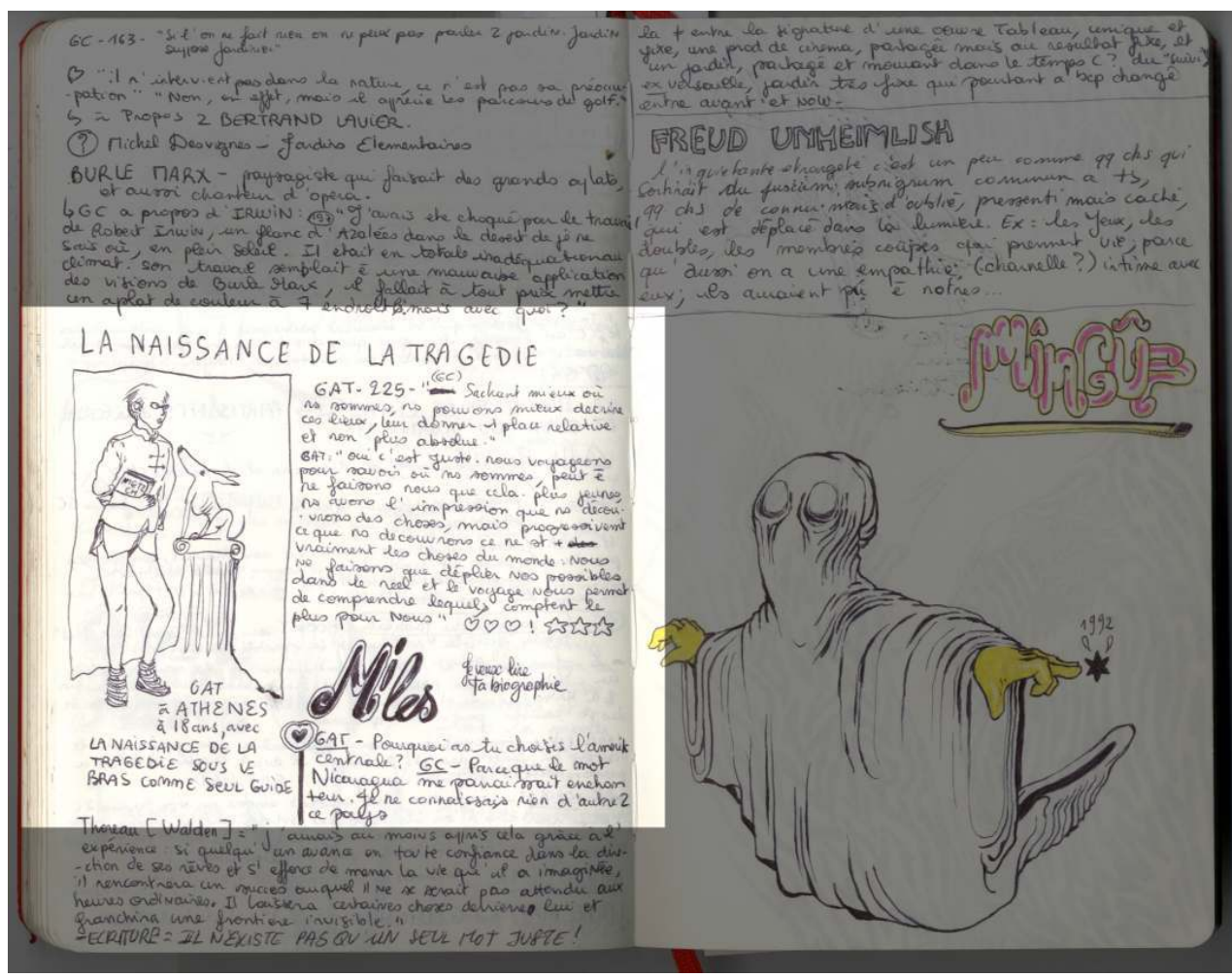

Fig. 8 : On voit ici que le texte s'est resserré (Gilles A. Tiberghien à Athènes, lisant La Naissance de la tragédie de Nietzche)

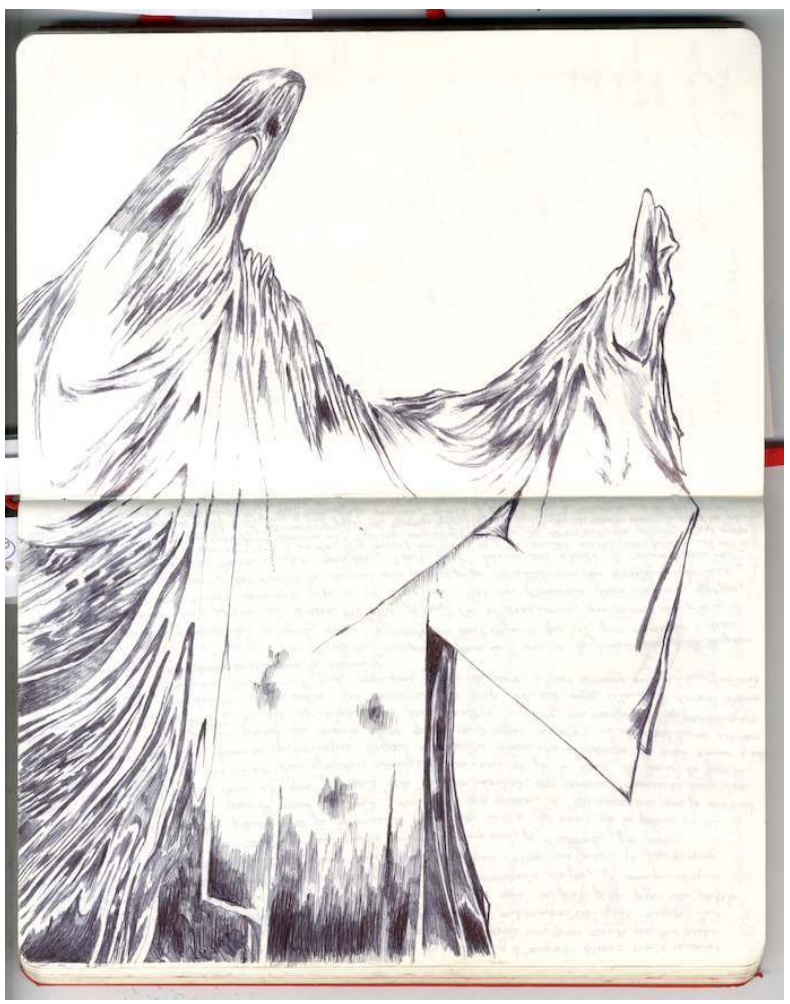

Fig. 9 : Première mise en place de ces enseignements : le livre de Deleuze, la façon dont j'ai transcrit ses idées dans une écriture minutieuse, utilisant plusieurs semaines le même Bic noir comme unique outil, et les croquis de plus en plus précis qui les accompagnent m'ont permit d'intégrer la pensée de Deleuze dans un trait et une écriture. La pensée a migré dans le trait. 


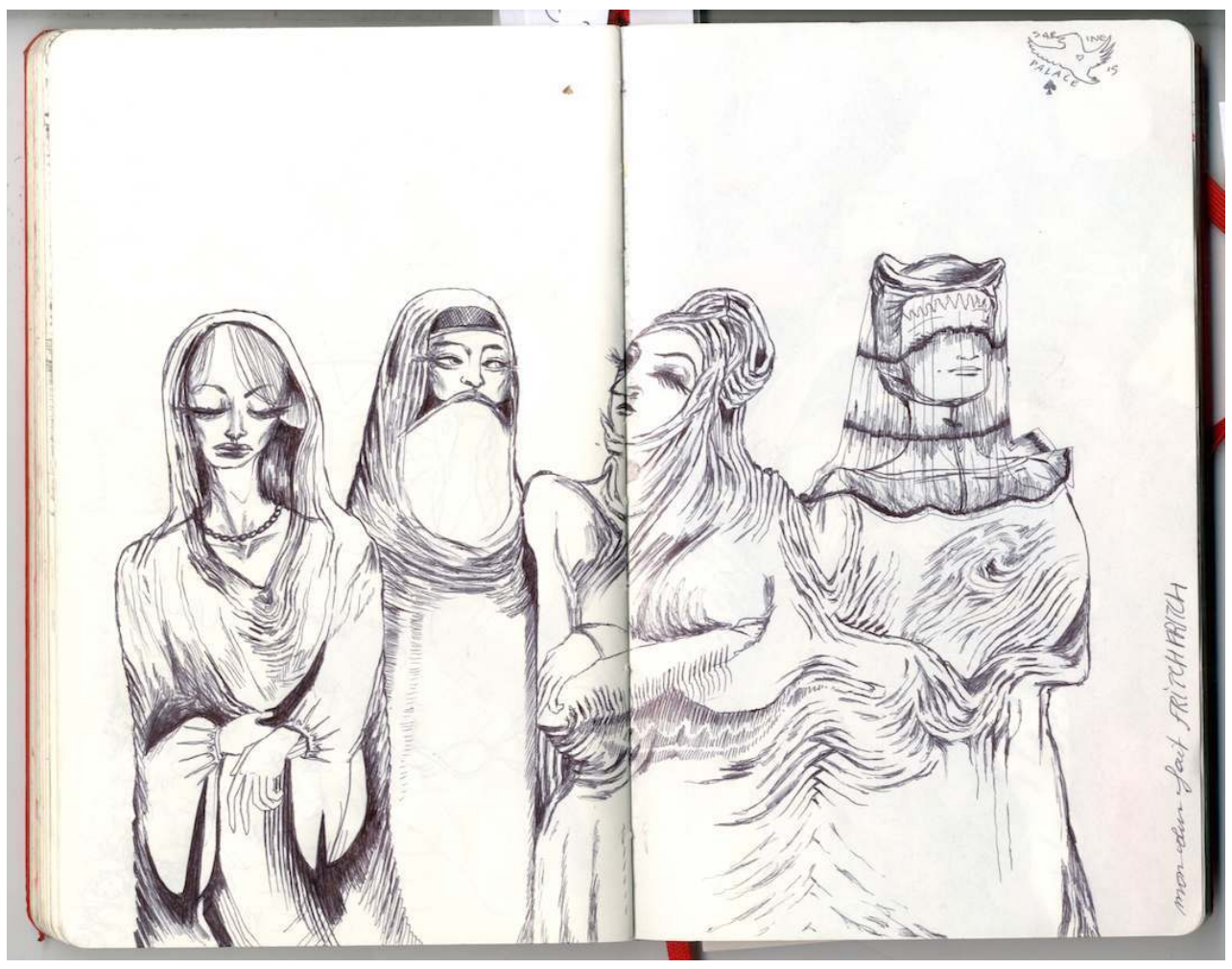

Fig. 10 : Quelques jours plus tard, deuxième dessin plus orienté encore sur le pli. On retrouve plusieurs exercices qui viennent directement de l'écriture dans l'utilisation des lignes comme diverses polices. Les traits utilisés ici expérimentent différentes textures et lignes, c'est un dessin plus abouti mais aussi un vrai terrain de recherches : quelle est la ligne la plus juste? II y a une sorte d'infusion de la pensée - encore très illustrative - dans ce dessin. J'ai dessiné des plis de tissu de façon très formelle, mais ils sont encore assez raturés et brouillons. II y a comme une métaphore dans le texte : une image qui renvoie à une idée. 


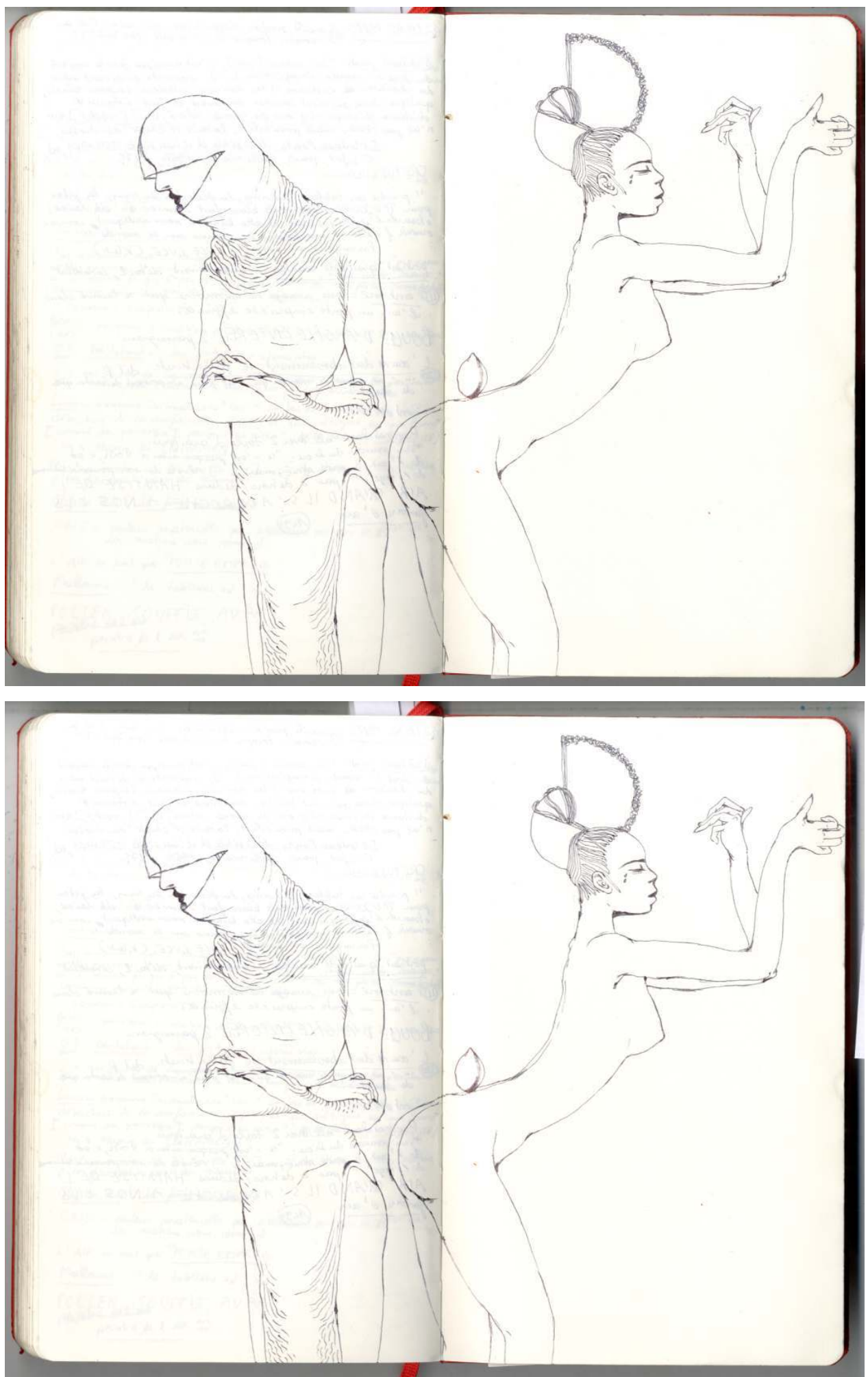

Fig. 11 : Les plis se font de plus en plus précis. Ce langage de trait s'intègre à mon dessin et se fond peu à peu dans des formes plus anciennes. 


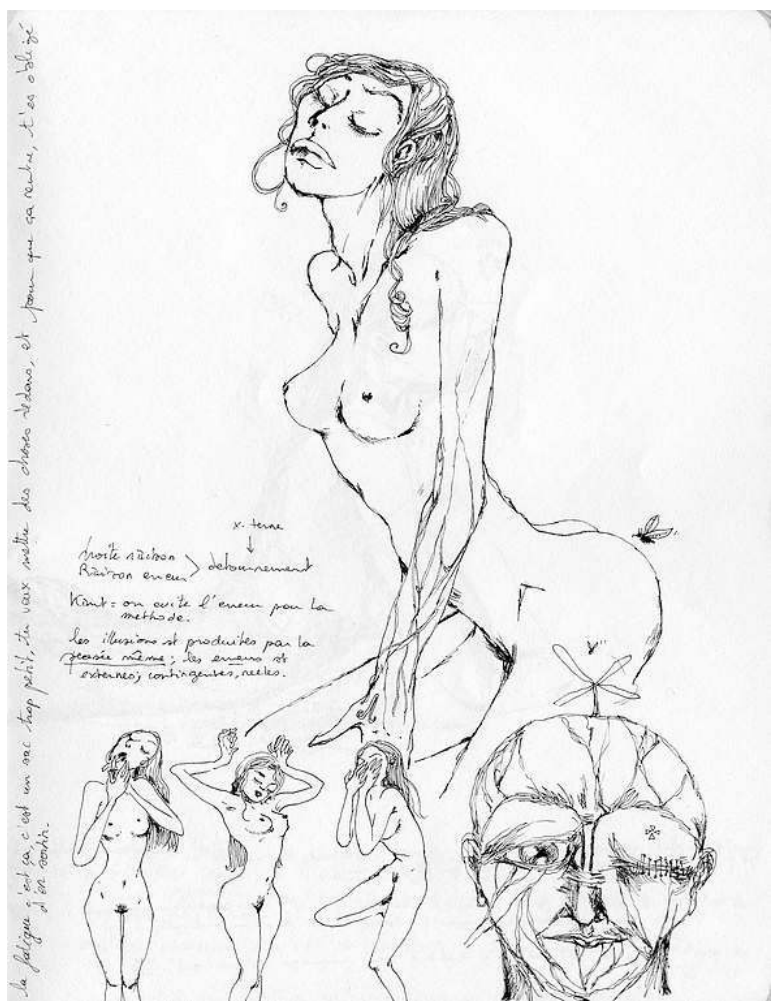

Fig. 12 : Dessin fait en cours de philosophie en 2014. II n'est peut-être pas anodin que les dessins associés à des pensées complexes soient plus précis, comme si une pensée aiguë provoquait en moi une attention accrue qui se traduit par une plus grande finesse dans la ligne d'écriture et de dessin.

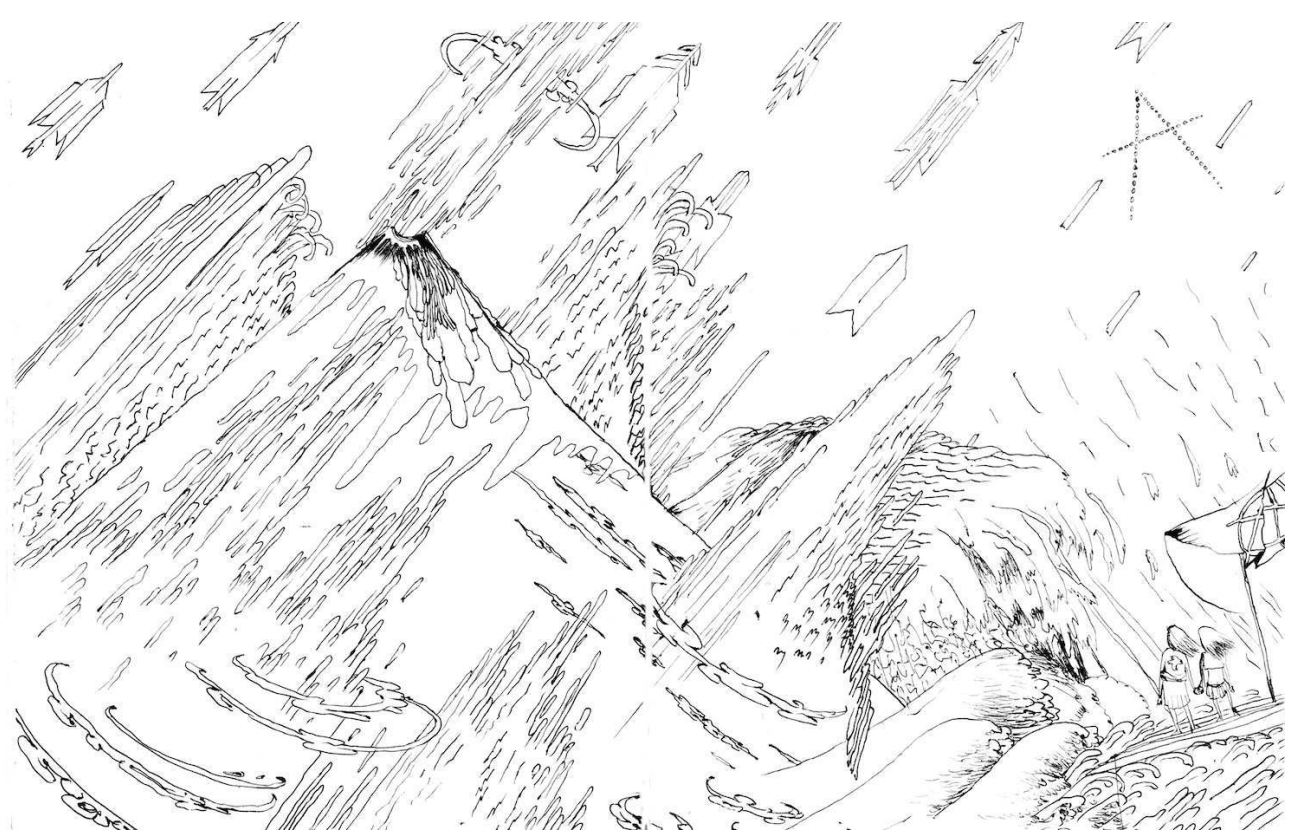

Fig. 13 : Cette ligne-pensée s'applique maintenant au paysage. 


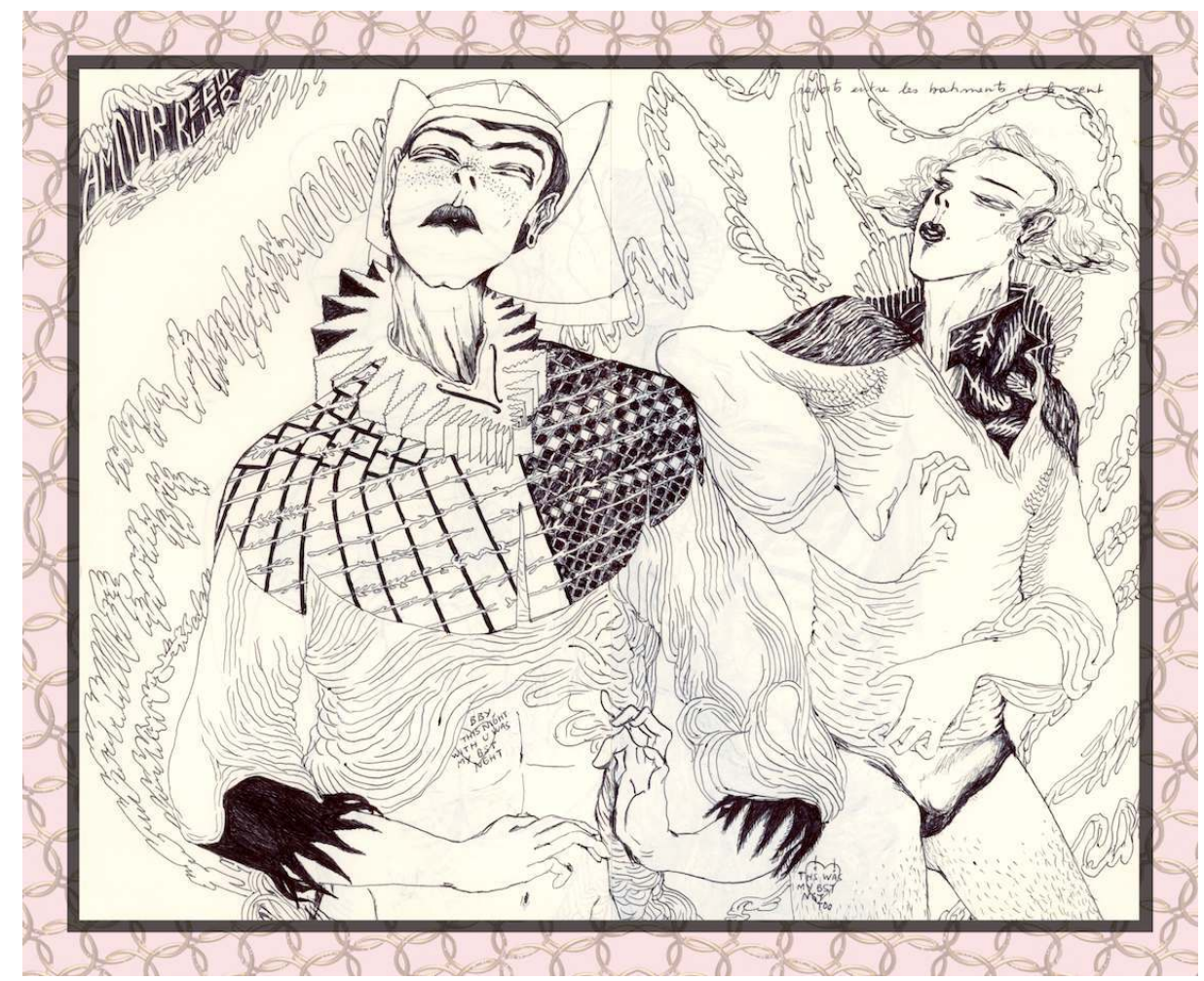

Fig. 14 : Cette pensée transformée en ligne est désormais profondément ancrée dans mon trait et se développe autour d'autres thèmes que la figure plastique du pli. Il reste cet aspect de drap froissé et cette précision inspiré des monades qui contiennent en elle la totalité de la création.

Le carnet, lorsqu'on le reprend, permet de retrouver la genèse d'une idée ou d'identifier d'où vient un trait graphique. En ce sens, il est vraiment une archéologie de la forme et de l'idée, et l'on peut la parcourir à l'envers comme une carotte glaciaire pour mieux comprendre le fondement de certaines habitudes de pensée ou de dessin. Il est aussi une réserve d'idées où l'on peut revenir puiser du matériel frais si on a l'esprit un peu sec. Refeuilleter ses carnets donne toujours l'impression d'une immensité de possibles qui n'ont pas étê explorés, de bribes d'idées oubliées, de flèches lancées en tous sens sans atteindre leur cible. Mais si tout n'est pas utilisé, on trouve souvent une certaine cohérence entre des références anciennes que l'on a enfin lues, ou simplement comme plus haut l'infusion d'un concept dans la ligne, par exemple. Le carnet est aussi un itinéraire croisé, des idées apparaissent par clignotement au fil des pages et finissent par devenir urgentes, et portées à la réalisation. Au cours de ce surgissement progressif au fil des pages, elles peuvent se frotter à tout ce qui traine sur la page, et c'est ainsi qu'un concept de Deleuze se voit augmenté d'une vision foucaldienne, après l'avoir trop longtemps fréquentée dans le voisinage du carnet. 


\section{NOTES}

1. Roland Barthes, «Cy Twombly, ou Non multa sed multum », dans L'Obvie et l'obtus, Paris, Éditions du Seuil, coll. » Points », 1992 [1976], p. 145-162.

2. Gilles Deleuze, Le Pli. Leibniz et le Baroque, Paris, Éditions de Minuit, 1988.

\section{RÉSUMÉS}

Sabine Teyssonneyre explore, au cœur de ses propres carnets d'esquisses et d'études, les degrés successifs d'un dégagement intérieur du trait à l'égard de tout ce qui pourrait trop fortement le conditionner et le rendre prévisible. La prégnance du support et des outils du tracé, choisis au terme d'un processus lié aux sensations du dessin, laisse progressivement la place à une disponibilité intellectuelle et sensuelle qui fait feu de toute rencontre, en l'occurrence philosophique. Se voit ainsi examiné, via la pensée du pli chez Deleuze, le "dépli » inattendu d'un concept qui se réalise en traits nouveaux et en figures de drapés fantastiques où naît un langage graphique.

Sabine Teyssonneyre uses her own sketchbooks to explore the successive degrees of an internal separation of the line from all that might condition it too strongly and make it foreseeable. The influence of the medium and the tools, selected after a process connected with the sensation of the drawing, gradually yield to an intellectual and sensual availability that makes the most of any encounter, in this instance philosophical. Using the concept of "pli" (fold) in Deleuze, she examines the unexpected "unfolding" of a concept that materializes in new lines and figures of fantastic draperies, giving rise to a graphic language.

Sabine Teyssonneyre explora -en el interior de sus propios cuadernos de esbozos y estudios- las etapas sucesivas de una liberación interior del trazo con respecto a todo lo que podría condicionarlo profundamente y volverlo previsible. La preponderancia del soporte y los instrumentos de trazado -escogidos después de un proceso vinculado con las sensaciones del dibujo- deja lugar progresivamente a una disponibilidad intelectual y sensual que saca provecho de todos los encuentros, en este caso, filosóficos. Esto permite estudiar -a partir de la noción de pliegue en Deleuze- el despliegue inesperado de un concepto que se realiza en nuevos trazos y en figuras de drapeados fantásticos de los que surge un lenguaje gráfico.

Sabine Teysonneyre esplora, nel cuore dei suoi taccuini di schizzi e studi, i gradi successivi di una liberazione interiore del tratto rispetto a tutto ciò che potrebbe condizionarlo eccessivamente e renderlo prevedibile. La pregnanza del supporto e degli strumenti del tratto, scelti al termine di un processo legato alle sensazioni del disegno, lascia progressivamente il posto ad una disponibilità intellettuale e sensuale che utilizza qualsiasi tipo di incontro, nella fattispecie un incontro filosofico. Si trova così esaminato, attraverso il pensiero della "piega" in Deleuze, lo "spiegarsi" inatteso di un concetto che si realizza in tratti nuovi e in figure di drappeggi fantastici, dove nasce un linguaggio grafico. 
Sabine Teyssonneyre untersucht, anhand ihrer eigenen Skizzen- und Studienhefte, die sukzessiven Grade einer inneren Befreiung vom Federstrich angesichts dessen, was ihn zu stark in eine bestimmte Richtung lenken und vorhersehbar machen könnte. Die Prägnanz einer Unterlage und der Werkzeuge der Zeichnung, ausgewählt nach einem Prozess, der mit den Empfindungen des Entwurfs verbunden ist, weicht nach und nach einer geistigen und sinnlichen Verfügbarkeit, die jede Begegnung im philosophischen Falle befeuert. So wird - Bezug nehmend auf den Gedanken der „Falte“ bei Deleuze - die unerwartete „Entfaltung“ eines Begriffs untersucht, der sich in neuen Linien und in Gestalt von phantastischen Faltungen realisiert, woraus eine graphische Sprache entsteht.

\section{AUTEUR}

\section{SABINE TEYSSONNEYRE}

Après un Diplôme national d'arts plastiques à l'École supérieure des beaux-arts de Montpellier, Sabine Teyssonneyre, qui publie sous le nom de Sabine Palace, étudie en Master 2 de bande dessinée à l'École européenne supérieure de l'image d'Angoulême. Elle pratique le dessin, la gravure et la bande dessinée. Dans ses travaux, on croise des nonnes parties en croisière pour l'espace, des miniatures persanes et des super-héros en combinaisons moulantes. Elle écrit aussi des articles pour le journal La Guillotine et publie avec le Collectif 100 têtes. Son mémoire de première année s'intitulait « Mondes et bande dessinée : une approche par les jardins » et analysait le concept de monde hétérotopique en bande dessinée. Nous sommes ici en prise directe avec l'élaboration des figures et des personnages, au fil quotidien de ses carnets, de ses lectures et des bifurcations graphiques qui ponctuent le travail d'élaboration. 